\title{
PLANE SOURCE OF CONCENTRATED MAGNETIC FIELD BASED ON SQUARE COILS
}

\author{
Sergey Roslyakov ${ }^{l}$, Vladimir Yakubov, Ksenia Zavyalova \\ Radiophysics Faculty, Tomsk State University, Tomsk, Russia
}

\begin{abstract}
You can see the method of the synthesis of planar sources of concentrated magnetic field. Method defines the current distribution in the plane, which is required to produce a concentrated magnetic field at a distance from the source. Carried out a numerical calculation of the current distribution to create a point field localization. It is shown that the field concentration is achieved by minimizing the level of low spatial frequencies due to antiphase currents in concentric circular coils. The resulting field distribution is extremely sensitive to the slightest changes in the current distribution, which creates difficulties for the practical implementation of the system.
\end{abstract}

\section{Introduction}

Variable magnetic fields have a high potential for application in the area of non-destructive quality control and inspection systems [1-8]. The disadvantages of this type of introscopy may include: the detection only of electrically conductive objects, the rapid decrease of the field because of the distance, a low resolution. These methods are used to diagnose irregularities in ferromagnetic and electrically conductive objects, as well as for detecting electrically conductive objects behind the dielectric barriers. Weak alternating magnetic fields penetrate through metal barriers and can detect objects behind them [1-8]. For the non-destructive testing of ferromagnetic materials is possible to use permanent magnetic fields. To increase the dynamic range of magnetic induction methods are applied differential magnetic field sensors [2, 4]. There are various combinations of sensors based on differential inductors. In the differential magnetic induction sensor coil sources are located with the receiving coil in a way that does not create induced currents in the receiving coil. For example, two source coils located near each other can be used, but only if source coils energized antiphase currents. The receiving coil which is placed between them cannot induce current, while a metal object appears in the medium and causing a violation of the balance between the fields of the source coils. For magnetic induction defectoscopy more preferable to use induction coils instead of the Hall sensors [3], because the coil allows obtaining higher level of signal-to-noise ratio. The transmission methods of magnetic induction tomography are being developing [8]. These methods are based on the analysis of amplitude and phase distortion of magnetic force lines which passing through the test electrically conductive medium. One of the major problems of the magnetic

\footnotetext{
${ }^{1}$ Corresponding author: serega_1994_0@mail.ru
} 
tomography is a nonlocalizability of magnetic field at a distance from the source. That leads to the deterioration of resolution of reconstituted tomographic images.

This work aims to create a concentrated source of the magnetic field, which will solve the problem of low resolution magnetic induction introscopic systems.

\section{System overview}

It is proposed to consider the scheme of measurements shown in Figure1. Let's consider the plane current distribution which creates a magnetic field in space. It is necessary to select such current distribution that will provide a concentration of the magnetic field in the horizontal plane at distance $h$ and around a single point. Let us assume that all the currents that exist on the plane XOY are vortex. Consequently, they can be represented as a sum of a plurality of elementary eddy currents of different amplitude. Accordingly, the field generated by the induction currents can be represented as the sum of elementary eddy current fields. Mostly we are interested in the z-component which is component of the magnetic induction vector field $B_{z}$.

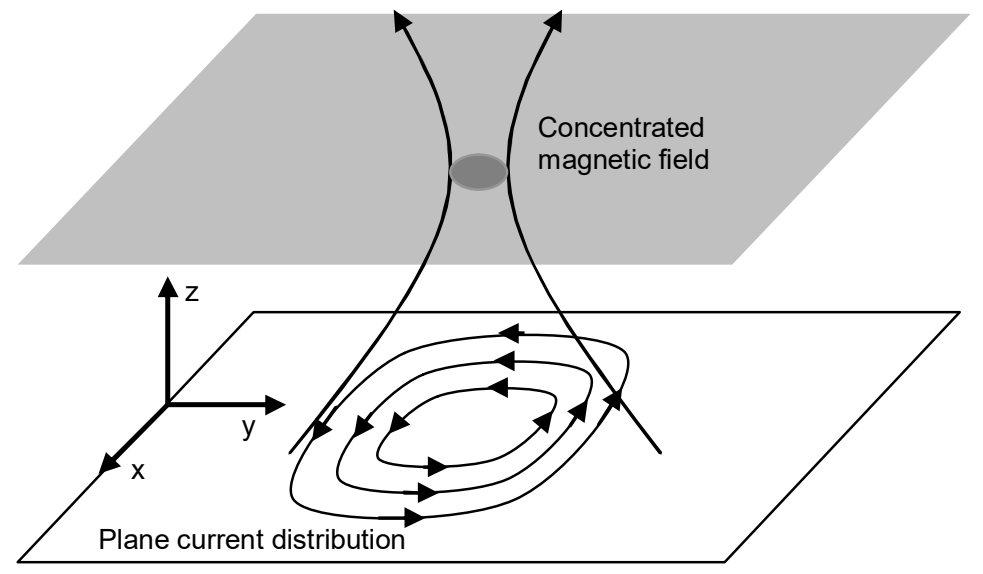

Fig.1. Measurement scheme.

Let us write the z-components of the magnetic induction vector field in the plane $z=h$ as an integral convolution:

$$
B_{z}(x, y, h)=\iint_{X O Y} p\left(x^{\prime}, y^{\prime}\right) S\left(x-x^{\prime}, y-y^{\prime}, h\right) d x^{\prime} d y^{\prime},
$$

where $S(x, y, h)$ - is the z-component of the magnetic induction field on $\mathrm{h}$ range and is created by elementary eddy current; $p\left(x^{\prime}, y^{\prime}\right)$ - distribution of eddy currents amplitude on the plane XOY. The field generated by the eddy current in the basic range of $10 \mathrm{~cm}$ is represented on Figure 2. It can be seen that the field is localized in the area of a diameter about $10 \mathrm{~cm}$. It can be seen that the field is localized to the area of a diameter of about 10 $\mathrm{cm}$. In fact, the resolution of magnetic induction sensing systems will deteriorate proportional to the distance. 


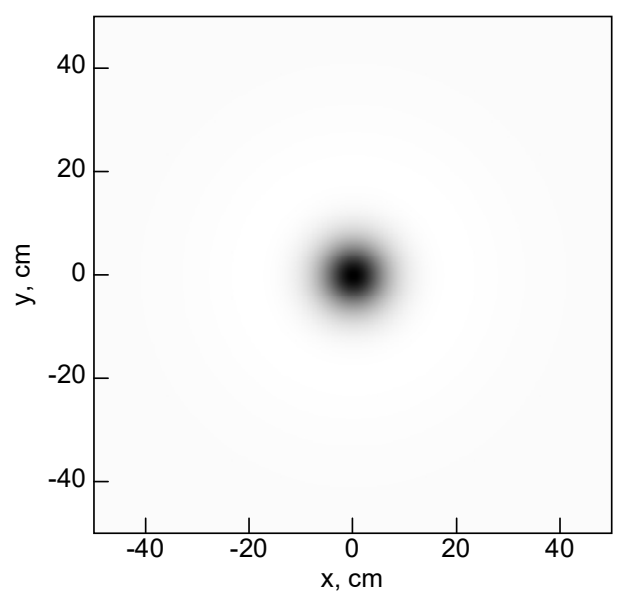

Fig.2. $B_{z}$ component of magnetic induction field of elementary eddy current.

\section{Solving the inverse problem}

It is necessary to choose such current distribution that provides the best concentration of the magnetic field at a distance $\mathrm{h}$ from the plane XOY. To solve this problem suggested submitting the required field distribution in the form of a delta function, and the current distribution can be found by using the operation of inverse convolution with regularization. The spatial spectrum of the current distribution in the plane is calculated from the spatial spectrum according to the expression:

$$
\tilde{p}\left(k_{x}, k_{y}\right)=\frac{\tilde{S}^{*}\left(k_{x}, k_{y}\right)}{\mid \widetilde{S}\left(k_{x}, k_{y}\right)^{2}+\alpha},
$$

где

$$
\tilde{p}\left(k_{x}, k_{y}\right)=\frac{1}{4 \pi^{2}} \int_{-\infty}^{\infty} \int_{-\infty}^{\infty} p(x, y) \exp \left(-i x k_{x}-i y k_{y}\right) d x d y
$$

$\widetilde{S}\left(k_{x}, k_{y}\right)=\frac{1}{4 \pi^{2}} \int_{-\infty}^{\infty} \int_{-\infty}^{\infty} S(x, y) \exp \left(-i x k_{x}-i y k_{y}\right) d x d y ; \alpha-$ parameter of regularization that is chosen to be sufficiently small to achieve a concentration of the field, but large enough to avoid the division into small quantities and cause an increase in the level of artifacts.

The seeking distribution of elementary eddy current is calculated using the Fourier transform of the calculated spatial spectrum:

$$
p(x, y)=\int_{-\infty}^{\infty} \int_{-\infty}^{\infty} \widetilde{p}\left(k_{x}, k_{y}\right) \exp \left(i x k_{x}+i y k_{y}\right) d x d y
$$

By means of formula (3) we have calculated the current distribution on the plane (Figure 3 ). In this case the current distribution is the convolution integral of $p(x, y)$ with elementary vortex function.

It can be seen that the resulting current distribution is axially symmetrical and looks like a set of ring currents which change direction on different diameters. Thus, currents at different diameters compensate each other field at low spatial frequencies. Due to the balance which was achieved there are only high spatial frequencies. This allows concentrating the field around a center point. 

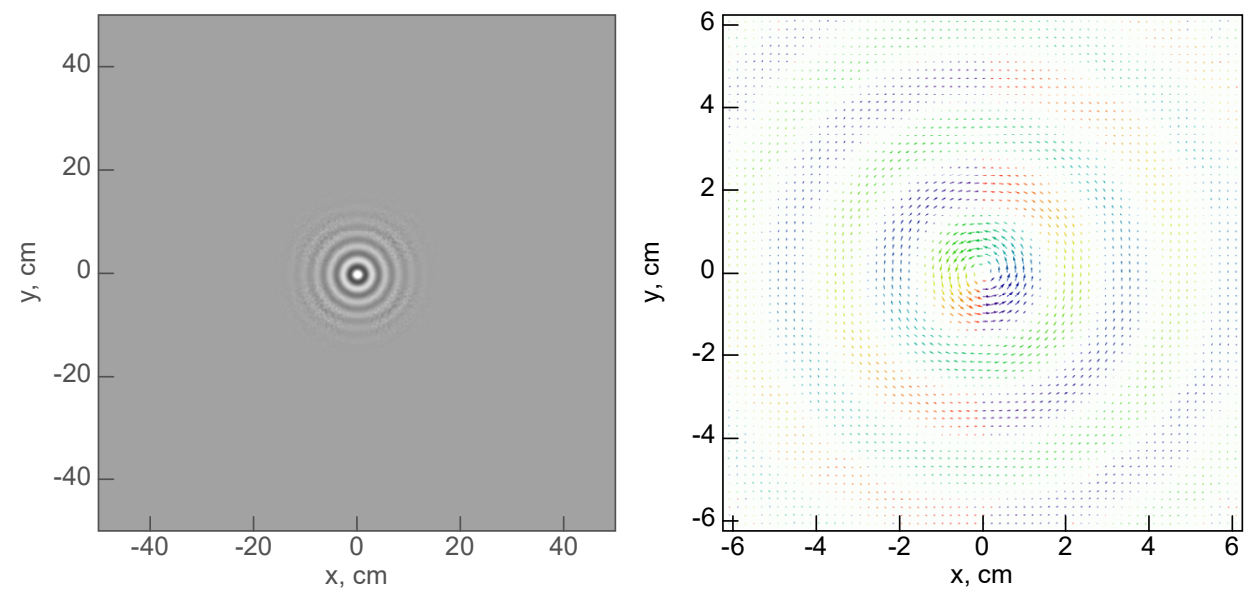

a

$\mathrm{b}$

Fig.3. Distribution of current to obtain concentrated magnetic field: a - distribution of elementary eddy currents; $b$ - distribution of place current density vector.

The Figure 4 shows the resulting field distribution which was calculated by using the formula (1) and (3). It can be seen that the field concentration reached 7 times stronger than the field concentration of elementary eddy current.

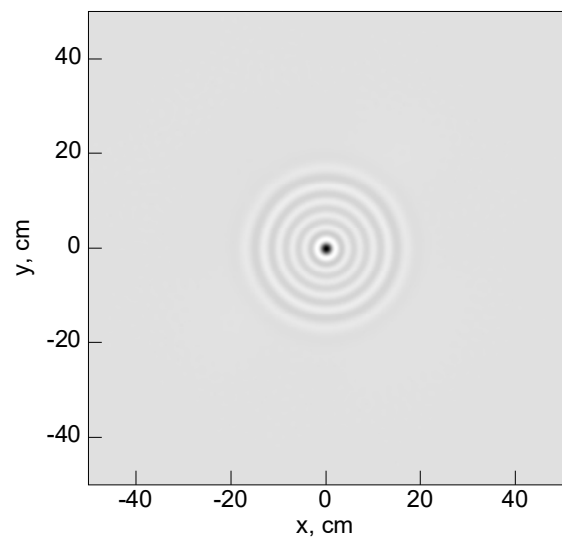

a

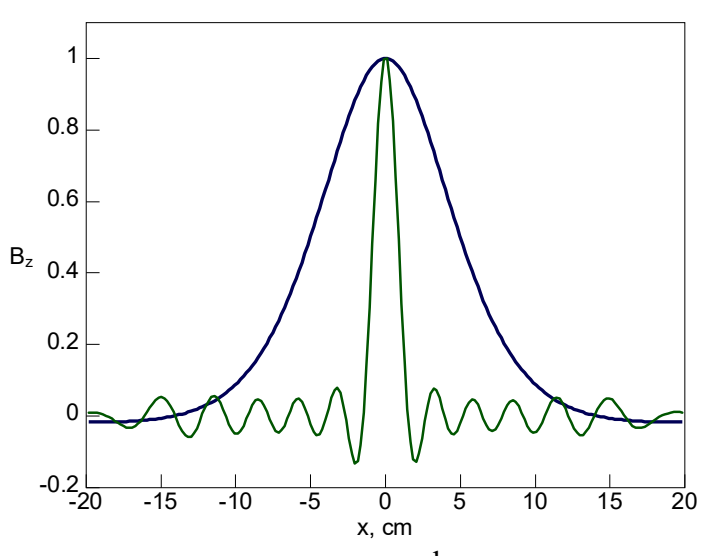

b

Fig.4. Distribution of z-component of magnetic induction field at a distance of $10 \mathrm{~cm}$ created by special current distribution: $\mathrm{a}$ - plane image of field; $\mathrm{b}$ - section of field (green) along $\mathrm{x}$ axis compared with the field of elementary eddy current (blue).

Should be noted that any distortion in the current distribution cause the disturbance of the balance and a sharp increase in low spatial frequencies in the magnetic field. This significantly impairs the concentration. This creates difficulties for the practical creation of such a power supply. It is known that if the wave field was focused that occurs multiple amplification of the field at the point of in-phase addition of the waves and at the concentration field. In this case, the concentration of the magnetic field is achieved through the suppression of low spatial frequencies, which significantly reduces the amplitude of the resulting field. However, there is a way to solve this problem. We can increase the amplitude of the current in the source.

The achieved effect is different from the focus, because it does not increase the amplitude of the field but rather reduces it repeatedly. The numerically calculated current 
distribution is extremely difficult to create physically. It is proposed to simplify the problem by considering only 3 square coils located on a plane. For each coil, current values will be selected to ensure the formation of a concentrated field.

We calculated the magnetic field of a system of three coils with a current. Numerical values were obtained for three square coils of different sides: $8 \mathrm{~cm}$, (current $1 \mathrm{~A}$ ), $11 \mathrm{~cm}$ (current $1.021 \mathrm{~A}$ ), $14 \mathrm{~cm}$ (current $0.306 \mathrm{~A}$ ). The result of modeling the field at a distance of $10 \mathrm{~cm}$ is presented in Figure 5.

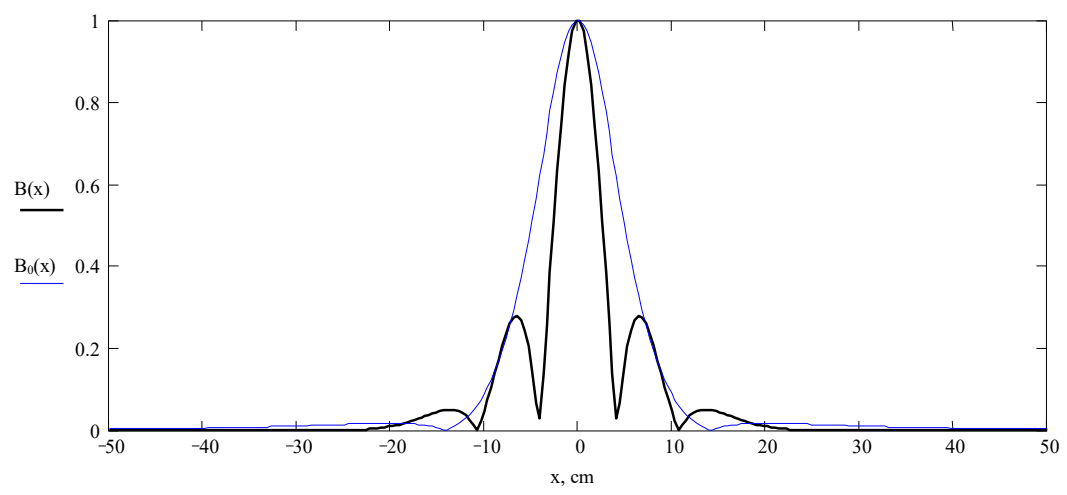

Fig.5. Magnetic field distribution at $10 \mathrm{~cm}$ distance.

It can be seen that the field concentration has broadened than for a continuous current distribution. In this case it is possible to reach the field concentration twice as much as for an elementary source. However, a similar configuration of the coils can be made practically.

\section{Conclusion}

Here is proposed a method of synthesis of current distribution which provides a concentration of the magnetic field at a distance. The concentration is achieved by reducing the level of low spatial frequencies of the field, while maintaining high spatial frequencies. The sources of a similar type field will be used in magnetic induction tomography of high resolution.

\section{Acknowledgment}

The research is supported by Russian Science Foundation project No 16-19-10272.

\section{References}

[1] A.A. Kolyshkin, R. Vaillancourt, Journal of Applied Physics. 77, 4909 (1995).

[2] S. Li, S. Huang, W. Zhao, Sensors and Actuators A. 135, 675 (2007)

[3] S. Li, S. Huang, W. Zhao, P. Yu. Sensors and Actuators A. 141, 13 (2008)

[4] X. Peng, H. Songling, Z. Wei, NDT\&E International. 44, 339 (2011)

[5] R.J. Ditchburn, S.K. Burke, NDT\&E International. 38, 690 (2005)

[6] J.O. Fava, M.C. Ruch, NDT\&E International 39, 414 (2006)

[7] T.P. Theodoulidis, E.E. Kriezis, NDT\&E International 35, 407 (2002)

[8] A. Korjenevsky, V. Cherepenin, S. Sapetsky, Physiol. Meas. 21(1), 89 (2000) 